\title{
Diagrammatic Perturbation Technique for a Quantum Dot Connected to Superconducting Leads
}

\author{
V. POKORNÝ $\tilde{r}^{a, *}, \mathrm{~V}$. JANIŠ ${ }^{a}$, T. NOVOTNÝ ${ }^{b}$ AND M. ŽONDA ${ }^{b}$ \\ ${ }^{a}$ Institute of Physics, Academy of Sciences of the Czech Republic, Na Slovance 2, 18221 Prague, Czech Republic \\ ${ }^{b}$ Faculty of Mathematics and Physics, Charles University in Prague, Ke Karlovu 5, 12116 Prague, Czech Republic \\ We study spectral properties of a quantum dot attached to two superconductors with nonzero phase differ- \\ ence. The system is described as a single-impurity Anderson model coupled to BCS superconducting leads. We \\ utilize diagrammatic perturbation expansion in the Coulomb interaction to capture relevant physical phenomena, \\ particularly the effect of the Coulomb interaction on the Andreev bound states present in the electronic spectrum. \\ Results of the Hartree-Fock and the random phase approximations at zero temperature are presented.
}

DOI: 10.12693/APhysPolA.126.352

PACS: 71.15.-m, 73.21.La, 74.50.+r

\section{Introduction}

Hybrid superconductor-quantum dot systems like carbon nanotubes coupled to superconducting electrodes have attracted much attention in recent years. They provide an almost ideal system where the interplay between superconducting order and electronic correlations can be studied in a controlled way.

Such systems can be modelled by a single-impurity Anderson model coupled to superconducting leads. The quantum dot is described by the local energy level $\varepsilon$ and the on-site Coulomb interaction $U$. The leads are described within the BCS theory by the superconducting gap $\Delta$ and the phase difference $\Phi=\Phi_{R}-\Phi_{L}$ between the right $(\mathrm{R})$ and the left $(\mathrm{L})$ electrode. Finally, the dotlead coupling is described by a hybridization function $\Gamma=2 \pi t^{2} \rho$, where $t$ is the hopping amplitude between the quantum dot and the lead and $\rho$ is the density of states in the leads. In order to obtain analytic results we choose the density of states constant and the hybridization function identical for both leads, $\Gamma_{R}=\Gamma_{L}=\Gamma$. For more details see Refs. [1] and [2].

This model has been studied by various methods used for correlated systems, including Hartree-Fock approximation (HFA), quantum Monte-Carlo simulations and numerical renormalization group. For an exhaustive review of the results, see Ref. [2]. Each of these methods suits better for different set of input parameters and together they provide us with a number of interesting results. One of them is the existence of Andreev bound states (ABS) within the superconducting gap. These states form due to multiple Andreev reflections on the opposite dot-lead interfaces. Another interesting and experimentally observed property is the $0-\pi$ transition in the Josephson current, flowing through the quantum

*corresponding author; e-mail: pokornyv@fzu.cz dot when changing the phase difference $\Phi$. It describes reversal of the sign of the supercurrent under certain conditions.

The purpose of this contribution is to present an advanced, systematic method to calculate spectral (oneparticle) properties of the quantum dot, based on the perturbation expansion in the Coulomb interaction. The summation technique used here is a generalization of the random phase approximation (RPA) and it is applicable in a wide range of the input parameters. The resulting spectral functions, normal and anomalous, are essential for the calculation of the Josephson current. A special attention is paid to ABS, since they give the dominant contribution to the supercurrent.
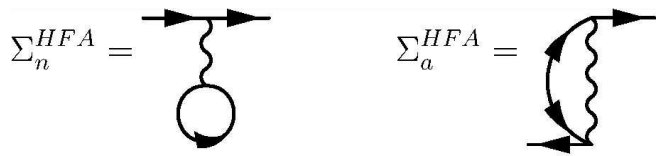

Fig. 1. Diagrammatic representation of the normal (left) and anomalous (right) self-energy in HFA. Wiggle line represents the Coulomb interaction $U$, line with single arrow is the normal propagator $G_{n}$, and line with double arrow is the anomalous propagator $G_{a}$ (this propagator is an even function of momentum, so it does not depend on its orientation).

\section{Hartree-Fock approximation}

We start our calculation from the Hartree-Fock approximation, representing the first order in the perturbation expansion. The Nambu spinor formalism is used to represent the impurity one-particle propagator $G\left(i \omega_{n}\right)$ as a $2 \times 2$ matrix. Here $\omega_{n}$ stands for the $n$-th fermionic Matsubara frequency $(2 n+1) \pi / \beta$ and $\beta$ is the inverse temperature. Diagonal elements represent the normal part of the propagator, which is important for calculation of the spectral properties. The off-diagonal elements are the anomalous ones and they are necessary for calculation of 
the Josephson current. Interaction effects from both the dot-lead coupling as well as from the Coulomb interaction are incorporated in the static self-energies $\Sigma$ that are graphically represented in Fig. 1. The self-energy is calculated self-consistently within this approximation and the HF propagators are obtained from the matrix form of the Dyson equation.

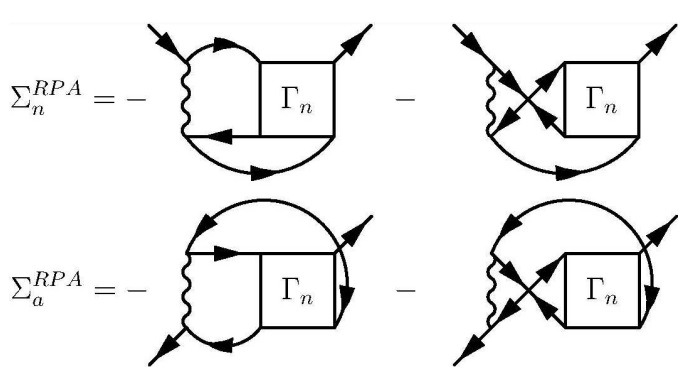

Fig. 2. Dynamic contribution (beyond HFA) to the normal (top) and anomalous (bottom) self-energy in RPA. $\Gamma_{n}$ is the two-particle vertex.

\section{Random phase approximation}

A straightforward way to go beyond HFA is the random phase approximation. It represents a reliable summation technique and it was successfully used to describe various phenomena in correlated systems, but has not yet been fully accomplished to the problem under investigation. The approximation leads to a dynamic (frequencydependent) self-energy which is obtained as a convolution of the particle propagators with the so-called two-particle vertex function $\Gamma_{n}\left(i \omega_{n}\right)$, plotted in Fig. 2. This vertex represents the sum of all multiple electron-hole scatterings (the ladder diagrams). For more details on this approximation, consult e.g. Ref. [3].

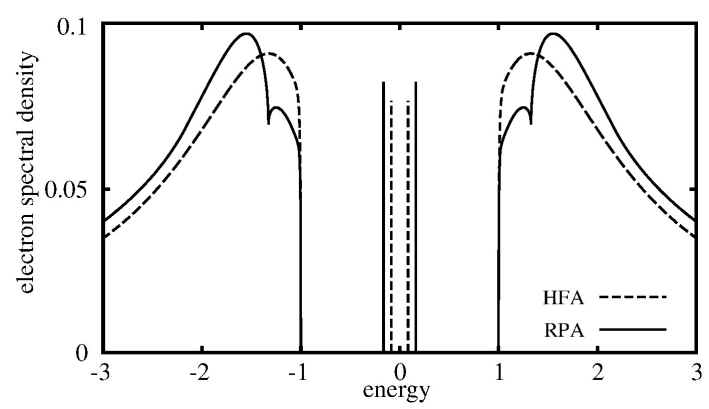

Fig. 3. Electron spectral density $-\operatorname{Im} G_{n} / \pi$ for the electron-hole symmetric case and $U / \Gamma=2, \Delta / \Gamma=1$, $\Phi=\pi / 2$. Dashed line: HFA results, full line: RPA results.

\section{Results and conclusions}

In Fig. 3 we plotted the zero-temperature electron spectral function $-\operatorname{Im} G_{n} / \pi$ and in Fig. 4 the current spectral function $-\operatorname{Im} G_{a} / \pi$ in the electron-hole symmetric case. We chose $U / \Gamma=2, \Delta / \Gamma=1$ and $\Phi=\pi / 2$ in

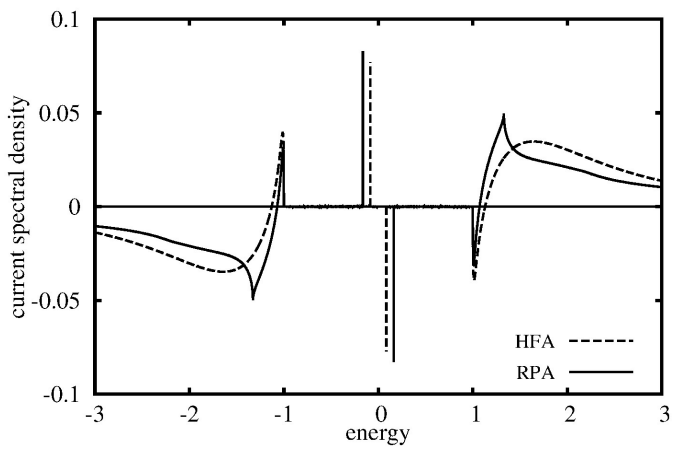

Fig. 4. Current spectral density $-\operatorname{Im} G_{a} / \pi$ for the same set of parameters as in Fig. 3 .

this calculation. These spectral functions were obtained by analytic continuation of the Matsubara propagators to the real frequency axis at zero temperature. This analytic continuation is straightforward. It is important in order to separate the contributions from ABS and from the continuous bands in the calculation of the Josephson current.

In the case of metallic leads $(\Delta=0)$ we would obtain the well-known three-peak structure in the RPA electron spectrum, known from the Fermi liquid theory. This serves us as a test of reliability of our calculations.

The width of the energy gap $2 \Delta$ is also preserved in both HFA and RPA. Since the determinant of the unperturbed matrix propagator diverges at $\pm \Delta$, no finite self-energy due to the Coulomb repulsion can change the positions of band edges.

The most important feature of the RPA spectra is the shift in the ABS positions w.r.t. HFA results. This can be considered as a footprint of possible crossing of the two ABS, which is related to the emergence of the $\pi$-junction behavior of the Josephson current.

\section{Acknowledgments}

This work was supported by the project P204/11/J042 of the Czech Science Agency. Support from project SVV 265301 of Charles University in Prague is also acknowledged.

\section{References}

[1] C. Karrasch, A. Oguri, V. Meden, Phys. Rev. B 77, 024517 (2008).

[2] A. Martín-Rodero, A. Levy Yeyati, Adv. Phys. 60, 899 (2011).

[3] A.C. Hewson, The Kondo Problem to Heavy Fermions, Cambridge Univ. Press, Cambridge (1993). 